\title{
Modelling of coupled heat and moisture flows around a buried electrical cable
}

\author{
Hossein Eslami $^{1}$, Olivier Cuisinier ${ }^{1, a}$, Farimah Masrouri $^{1}$ \\ ${ }^{1}$ Laboratoire d'Energétique et de Mécanique Théorique et Appliquée - UMR 7563 CNRS / Université de Lorraine - 2, Rue M. \\ Roubault, TSA 70605, 54501, Vandœuvre-lès-Nancy, France
}

\begin{abstract}
The admissible current within a buried electrical power cable is limited by the maximum allowed temperature of the cable (Joule effect). The thermal properties of the surrounding soil controls heat dissipation around the cable. The main focus of the study was to evaluate the coupled heat and moisture flow around such buried electrical cables. The heat dissipation of a buried power cable was simulated in the surrounding soil at unsteady conditions. The hydro-thermal coupling was modelled by taking into account the moisture flow of liquid water and vapour, and the heat flow in the soil by convection and advection. As the thermal vapour diffusion enhancement factor $(\eta)$ appears to be a key parameter, the sensitivity study of the coupled heat and moisture flow in the ground regarding this parameter was performed. The variations of the degree of saturation and the temperature of the surrounding soil were studied over 180 days of heating. The results showed that the moisture flow was mainly caused by the vapour transport under temperature gradients. These results emphasized the significant effect of the hydrothermal characteristics of surrounding soil. The radius of influence of the power cable was also evaluated.
\end{abstract}

\section{Introduction}

Nowadays, new electrical power lines are generally buried to limit their impact on landscape. When a buried electrical power cable passes the current, the conductor heats and releases calories (Joule effect). The admissible current of buried electrical cables is limited by the maximum allowed temperature of the cable. Low thermal conductivity prevents heat dissipation in the ground, and thus the cable temperature increases. It is essential to ensure effective dissipation of this energy to avoid excessive heating of the cable that could lead to its rupture.

The study of the heat dissipated from the cable in the surrounding soil has been the subject of many works [110]. Previous works focused on the thermal behaviour of cable backfill materials, the ampacity of cables, the effect of the installation geometry including the dimensions of the trench, cable location and diameter, the thermal properties of the surrounding soils, the seasonal variation of temperature, etc. Nevertheless, in our knowledge the coupling between thermal and hydraulic phenomena was not considered. The moisture flow induced by thermal gradient and the effect of water content on the thermal conductivity have to be taken into account to evaluate the coupled heat and moisture flow around such buried cables.

Philip and De Vries [11] investigated the coupling between liquid water, water vapour and heat transport in soils. They accounted for the fluxes induced by both pressure head and soil temperature gradients. The water vapour flux under thermal gradients is an important part of water flow in soil. Philip and De Vries [11] also added a mechanistic enhancement factor $(\eta)$ into the equation of thermally induced water vapour flow to consider the discrepancy between the predicted transfers using Fick's law and the measured water fluxes $[12,13]$. Despite the importance of $\eta$ in modelling of coupled heat and moisture flow, few works have been focused on evaluating it experimentally and quantitatively [14-17]. The available results showed that this parameter is not a constant and depends on water content and temperature variation. Other authors proposed empirical modifications for this parameter to improve its agreement with the observed experimental measurements $[18,19]$.

In this study, the heating of a buried electrical cable was simulated. The hydrothermal coupling was modelled taking into account the moisture flow in the form of liquid water and vapour, and the heat flow by convection and advection by the mean of PLAXIS $\AA$. The objective was to quantify the effect of the thermal vapour diffusion enhancement factor (mechanistic enhancement factor), $\eta$, on the thermal and hydraulic behaviour of the surrounding soil. The theory is first introduced. Then the numerical modelling will be described. The effects of $\eta$ is then discussed in a last section.

\footnotetext{
a Corresponding author: Olivier.Cuisinier@ensg.univ-lorraine.fr
} 


\section{Theory}

The moisture transfer in unsaturated soils takes place in two phases: liquid water and water vapour. The liquid water flow in unsaturated soils can be described using Darcy's law by replacing the saturated permeability with a relative permeability:

$$
\begin{aligned}
& \underline{J}_{w}=\rho_{w} \underline{v}_{w}=\rho_{w} \frac{k_{r e l}}{\mu} K \nabla\left(\rho_{w} g y+p_{w}\right) \\
& =\rho_{w} \frac{k_{r e l}}{\mu} K\left(\rho_{w} g+\nabla p_{w}\right)
\end{aligned}
$$

Where $J_{w}$ is the flux rate of mass of liquid water $\left(\mathrm{kg} / \mathrm{m}^{2} / \mathrm{s}\right), \rho_{w}$ is the density of water $\left(\mathrm{kg} / \mathrm{m}^{3}\right), v_{w}$ is the flow rate of water $(\mathrm{m} / \mathrm{s}), k_{\text {rel }}$ is the ratio of the permeability at a given saturation to the permeability in saturated state $(-), \mu$ is the dynamic viscosity of the water (N.s $\left./ \mathrm{m}^{2}\right), K$ is the intrinsic permeability of the soil $\left(\mathrm{m}^{2}\right), \mathrm{g}$ is the gravitational acceleration $\left(\mathrm{m}^{2} / \mathrm{s}\right)$, y is the elevation (m) and $p_{w}$ is the pore water pressure $\left(\mathrm{N} / \mathrm{m}^{2}\right)$.

The diffusion of water vapour through the soil, based on Fick's law can be written [11]:

$$
\underline{J}_{v}=-a \alpha D \nabla \rho_{v}
$$

where $J_{v}$ is the flux rate of mass of water vapour $\left(\mathrm{kg} / \mathrm{m}^{2} / \mathrm{s}\right), a$ is the air-filled porosity $\left(\mathrm{m}^{3} / \mathrm{m}^{3}\right), \alpha$ is the tortuosity (-), $D$ is the diffusivity of water vapour in air, $\left(\mathrm{m}^{2} / \mathrm{s}\right)$ and $\rho_{v}$ is the density of water vapour $\left(\mathrm{kg} / \mathrm{m}^{3}\right)$.

The water vapour flow can be expressed as:

$$
\begin{aligned}
& \underline{J}_{v}=-D_{v} \nabla \rho_{v}=-D_{v}\left(\begin{array}{l}
\eta\left(\theta \frac{\partial \rho_{v S}}{\partial T}+\frac{\rho_{v} p_{w}}{\rho_{w} R T^{2}}\right) \nabla T \\
+\left(\frac{-\rho_{v} p_{w}}{\rho_{w} R T}\right) \nabla p_{w}
\end{array}\right)= \\
& -\eta D_{v}\left(\theta \frac{\partial \rho_{v S}}{\partial T}+\frac{\rho_{v} p_{w}}{\rho_{w} R T^{2}}\right) \nabla T-D_{v}\left(\frac{-\rho_{v} p_{w}}{\rho_{w} R T}\right) \nabla p_{w} \Rightarrow \\
& \underline{J}_{v}=-D_{T v} \nabla T+D_{p v} \nabla p_{w}
\end{aligned}
$$

Where $\rho_{v S}$ is the saturated water vapour density $\left(\mathrm{kg} / \mathrm{m}^{3}\right)$ and $h_{r}$ is the relative humidity $(-), T$ is the Temperature $(\mathrm{K}), \mathrm{R}$ is the specific gas constant for water vapour, $D_{p v}$ is the hydraulic vapour diffusion coefficient, $D_{T v}$ is the thermal vapour diffusion coefficient and $\eta$ is the thermal vapour diffusion enhancement factor.

The water mass balance is given by this equation:

$$
\begin{aligned}
& n \frac{\partial}{\partial t}\left(S \rho_{w}+(1-S) \rho_{v}\right)+\left(S \rho_{w}+(1-S) \rho_{v}\right)\left[\frac{\partial \varepsilon_{v}}{\partial t}+\frac{1-n}{\rho_{s}} \frac{\partial \rho_{s}}{\partial t}\right] \\
& =-\nabla \cdot\left(\underline{J}_{w}+\underline{J}_{v}\right)
\end{aligned}
$$

Where $n$ is the porosity of porous medium, $S$ is the degree of saturation, $\rho_{s}$ is the soil grains density $\left(\mathrm{kg} / \mathrm{m}^{3}\right)$ and $\varepsilon_{v}$ is the volumetric strain. After expansion of the lefthand side of this equation by integrating the compressibility and volumetric thermal expansion of water and the volumetric thermal expansion of soil grains, the water mass balance can be expressed as [20]:

$$
\begin{aligned}
& {\left[n\left(\rho_{w}-\rho_{v}\right) \frac{\partial S}{\partial p_{w}}-n S \rho_{w} \beta_{w P 0}-n(1-S) \frac{\rho_{v}}{\rho_{w} R_{v} T}\right] \frac{\partial p_{w}}{\partial t}+} \\
& {\left[n\left(\rho_{w}-\rho_{v}\right) \frac{\partial S}{\partial T}-n S \rho_{w} \beta_{w T O}-n(1-S)\left(\theta \frac{\partial \rho_{v S}}{\partial T}+\frac{\rho_{v} \rho_{w}}{\rho_{w} R_{v} T^{2}}\right)\right] \frac{\partial T}{\partial t}+} \\
& {\left[\left(S \rho_{w}+(1-S) \rho_{v}\right)(1-n) \beta_{s T}\right] \frac{\partial T}{\partial t}+(S \rho w+(1-S) \rho v) \frac{\partial \varepsilon v}{\partial t}+} \\
& \nabla .\left(\underline{J}_{w}+\underline{J}_{v}\right)=0
\end{aligned}
$$

Where $\beta_{w P 0}, \beta_{w T 0}$ are the compressibility and volumetric thermal expansion of water and $\beta_{s T}$ is the volumetric thermal expansion of soil grains.

The heat flow by conduction is governed by Fourier's law:

$$
\underline{J}_{c}=-\lambda \nabla T
$$

The thermal conductivity of the porous medium, $\lambda$, could be expressed as:

$$
\lambda=(1-n) \lambda_{s}+n S \lambda_{w}+n(1-S) \lambda_{v}
$$

Where $\lambda_{s}, \lambda_{w}, \lambda_{v}$, are the solid, the water and the vapour thermal conductivities. By calculating the thermal conductivity of the porous medium with the equation (7), the effect of the degree of saturation variation (e.g. soil drying) on this parameter is taken into account.

Heat flow by advection is given by:

$$
\underline{J}_{A w}=C_{w} T \underline{J}_{w}=C_{w} T \rho_{w}\left(\frac{k_{r e l}}{\mu} \underline{\underline{\kappa^{\mathrm{int}}}}\left(\nabla p_{w}+\rho_{w} \underline{g)}\right)\right.
$$

Where $C_{w}$ is the water specific heat capacity

The energy balance equation for the porous medium can be written as follows:

$$
\begin{aligned}
& \frac{\partial T}{\partial t}\left(n S \rho_{w} e_{w}+n(1-S) \rho_{v} e_{v}+(1-n) \rho_{s} e_{s}=\right. \\
& -\nabla \cdot\left(\underline{J}_{A w}+\underline{J}_{c}\right)+Q_{T}
\end{aligned}
$$

Where $e_{w}, e_{v}$ and $e_{s}$ are the internal energy in the water, vapour and solid phases, respectively and $Q_{T}$ is the heat source term (i.e. heat generation rate per unit volume). The left-hand side of this equation could be replaced by $\rho C \frac{\partial T}{\partial t}$, where $\rho C$, the heat capacity of the porous medium is equal to:

$$
\rho C=(1-n) \rho_{s} C_{s}+n S \rho_{w} C_{w}+n(1-S) \rho_{v} C_{v}
$$

Where $C_{s}, C_{w}$ and $C_{c}$ are the solid, the water and the gas specific heat capacities.

Expanding the right-hand side of the equation (9) and adding a term to take into account the heat transfer at the surface in contact with the air, the heat balance equation can be written as:

$$
\begin{aligned}
& \rho C \frac{\partial T}{\partial t}-\nabla \cdot(\lambda \nabla T)+\rho_{w} C_{w}\left[\frac{k_{r e l}}{\mu} \underline{\underline{K^{\mathrm{int}}}}\left(\nabla p_{w}+\rho_{w} \underline{g}\right)\right] \cdot \nabla T \\
& +\rho_{w} C_{w} T\left[\nabla \cdot\left(\frac{k_{r e l}}{\mu} \underline{\underline{\kappa^{\mathrm{int}}}}\left(\nabla p_{w}+\rho_{w} \underline{g}\right)\right)\right]-Q_{T}-C_{a s}\left(T-T_{a}\right)=0
\end{aligned}
$$


Where $T_{a}$ is the air temperature $(K)$ and $C_{a s}\left(\mathrm{~W} / \mathrm{m}^{2} \mathrm{~K}\right)$ is the convective heat transfer coefficient at the surface in contact with air.

\section{Numerical modelling}

The equations described is previous section have been implemented and released in the finite element code PLAXIS 2D [20]. Thermal module of this code offers a coupling between the thermal phenomena. This code was used to simulate the hydrothermal behaviour of a trench and the surrounding soil when the power cables were the heat sources. In this section, first the modelled trench is described. Then the different material characteristics are presented and the boundary conditions are explained.

\subsection{Model description}

To study the heating of the soil surrounding a buried electrical cable, it was decided to model the standard configuration for a typical buried electrical system. The trench is $1.50 \mathrm{~m}$ in depth and $0.60 \mathrm{~m}$ in width. Threephase electric power cables are buried next to each other. The diameter of the cable is $86.6 \mathrm{~mm}$. Concrete is installed around the cables to ensure mechanical stability of the cables after installation. Compacted soil is then installed above the concrete. Most of the time, the trenches are done under existing road, the top material of the trench was thus an asphaltic layer. Figure 1 shows the sketch of the trench in the surrounding soil, which was modelled in the framework of this study. Thus 4 materials, plus the cables, were defined:

- material 1: asphaltic concrete + base,

- material 2: sub-base,

- material 3: concrete,

- material 4: surrounding soil.

Thanks to the symmetry, the modelling was done only for the half of the trench relative to the $\mathrm{Y}$ axis. The mesh was automatically generated and the geometry was divided into 15-Node triangular elements with a refinement in the trench. Figure 1 shows the mesh that contains 1987 nodes and 16199 elements. The results of the preliminary studies showed that for the dimensions equal to $10 \mathrm{~m}$, the scale effect is negligible.

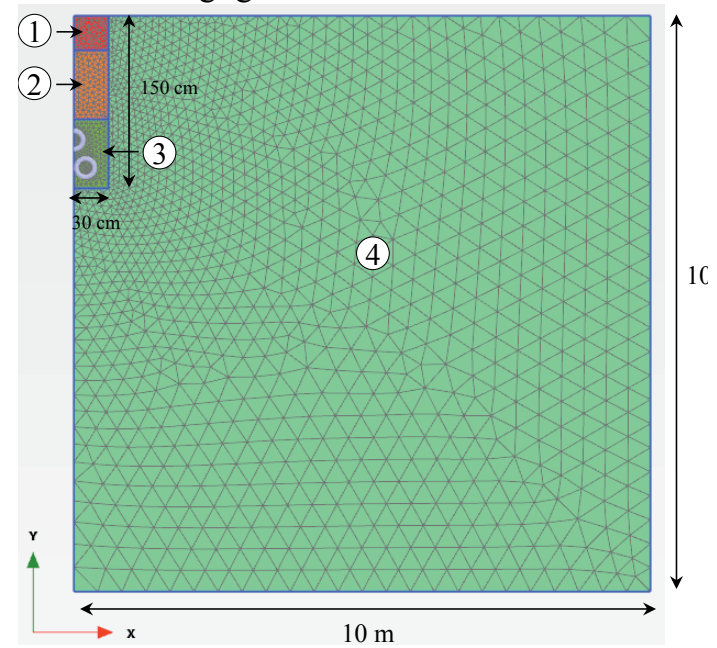

Figure 1. Geometry and meshing of the modelled trench.

\subsection{Material properties}

The thermal, hydraulic and hydrothermal properties of the surrounding soil are presented in Table 1. For the surrounding soil, a silt loam clay, the Van Genuchten model was used for the soil water retention curve.

Table 1. Thermal properties of the materials of the surrounding soil.

\begin{tabular}{|ccc|}
\hline \multicolumn{2}{|c|}{ Property } & Value \\
\hline Thermal & $C_{s}(\mathrm{~kJ} / \mathrm{t} . \mathrm{K})$ & 2000 \\
& $\lambda_{s}(\mathrm{~W} / \mathrm{m} . \mathrm{K})$ & 2.5 \\
& $\rho_{s}(\mathrm{~kg} / \mathrm{m} 3)$ & 2700 \\
\hline Hydraulic & $\left(D_{v}\right)(\mathrm{m} 2 /$ day $)$ & 0.1033 \\
& $\eta$ & 1 and 5 \\
\hline Hydraulic & $\mathrm{k}_{\mathrm{x}}=\mathrm{k}_{\mathrm{y}}(\mathrm{m} /$ day $)$ & 0.1676 \\
& $\mathrm{~S}_{\mathrm{res}}(-)$ & 0.06203 \\
& $\mathrm{~S}_{\mathrm{sat}}(-)$ & 1.00 \\
& $\mathrm{~g}_{\mathrm{n}}(-)$ & 1.377 \\
& $\mathrm{~g}_{\mathrm{a}}(-)$ & 3.83 \\
& $\mathrm{~g}_{\mathrm{l}}(-)$ & 1.25 \\
\hline
\end{tabular}

The diffusivity of water vapour $\left(D_{v}\right)$ is obtained with:

$$
D_{v}=a \alpha D
$$

Where $a$ is the air-filled porosity $\left(\mathrm{m}^{3} / \mathrm{m}^{3}\right), \alpha$ is the tortuosity (-) and $D$ is the diffusivity of water vapour in air, $\left(\mathrm{m}^{2} / \mathrm{s}\right)$. The tortuosity $(\alpha)$ is described as a function of the volumetric water content $(\theta)$ [21] with:

$$
\alpha=(n-\theta)^{2 / 3}
$$

Therefore, for a soil with the porosity $(n)=0.3$ and the air-filled porosity $(a)=0.15$ and the volumetric water content $(\theta)=0.15$, the tortuosity $(\alpha)$ was equal to: $\alpha=(0.3-0.15)^{2 / 3}=0.28$

The diffusivity of water vapour in air $(D)$ is expressed as a function of temperature (Kelvin) [22] with:

$$
D=2.29 \times 10^{-5}(T / 273.15)^{1.75}
$$

Assuming that the soil temperature changes between 12 and $60{ }^{\circ} \mathrm{C}$ (i.e. the maximum allowed temperature of the cable) the mean temperature was $36{ }^{\circ} \mathrm{C}$ and the diffusivity of water vapour in air is:

$D=2.29 \times 10^{-5}(309.15 / 273.15)^{1.75}=2.84 \times 10^{-5} \mathrm{~m}^{2} / \mathrm{s}=2.46 \mathrm{~m}^{2} /$ day The diffusivity of water vapour is:

$D_{v}=a \alpha D=0.15 \times 0.28 \times 2.46=0.1033 \mathrm{~m}^{2} /$ jour.

Thermal vapour diffusion enhancement factor, $\eta$, varies between about 1 and 15 depending on the volumetric water content and temperature [14, 23-25]. 
This dependence was not considered explicitly by the model. A constant value of $\eta$ was thus set for each simulation. To account for the dependency of $\eta$ on water content, the value of $\eta$ was assumed equal to 1 and 5 corresponding to dry and moderately humid soil initial conditions respectively. Using the same initial degree of saturation permitted to study explicitly the effect of $\eta$ on the results.

The thermal properties of the different materials of the trench are presented in Table 2. The hydraulic properties of these materials were not taken into account in order to focus the study on the hydrothermal behaviour of the surrounding soil.

Table 2. Thermal properties of the materials of the trench.

\begin{tabular}{|lccc|}
\hline Property & $\begin{array}{c}\text { Material 1: } \\
\text { asphaltic } \\
\text { concrete }+ \\
\text { base }\end{array}$ & $\begin{array}{c}\text { Material 2: } \\
\text { sub-base }\end{array}$ & $\begin{array}{c}\text { Material 3: } \\
\text { concrete }\end{array}$ \\
\hline$C_{s}(\mathrm{~kJ} / \mathrm{t} . \mathrm{K})$ & 960 & 1500 & 900 \\
$\lambda_{s}(\mathrm{~W} / \mathrm{m} . \mathrm{K})$ & 1.95 & 1 & 1.2 \\
\hline
\end{tabular}

\subsection{Initial and boundary conditions}

The initial conditions consisted of a homogeneous temperature of $12{ }^{\circ} \mathrm{C}\left(285.15^{\circ} \mathrm{K}\right)$ and the water table depth at $4 \mathrm{~m}$ below the surface (Figure 2). The boundary conditions (hydraulic and thermal) were applied to each side of the model. Along the axe OY, the water flux was closed (Neumann boundary condition) and the temperature could change (Dirichlet boundary condition). For the right side boundary, the water and heat fluxes were open. For the bottom, the water flux was allowed and a constant temperature was imposed $\left(285.15^{\circ} \mathrm{K}\right)$. For the top boundary, the heat transfer at the surface in contact with the air was allowded by imposing the air temperature equal to $20{ }^{\circ} \mathrm{C}\left(293.15^{\circ} \mathrm{K}\right)$ and defining $C_{a s}$ $\left(\mathrm{W} / \mathrm{m}^{2} \mathrm{~K}\right)$, the convective heat transfer coefficient at the surface in contact with air [26] (convective boundary condition):

$$
C_{a s}=7.2+3.8 v
$$

Where $v$ is the wind speed $(\mathrm{m} / \mathrm{s})$. The wind speed was considered equal to $20 \mathrm{~km} / \mathrm{h}(=5.56 \mathrm{~m} / \mathrm{s})$ as reference, $C_{a s}$ was:

$$
C_{a s}=7.2+3.8 \times 5.56=28 \mathrm{~W} / \mathrm{m}^{2} \mathrm{~K}
$$

The evaporation in the interface of soil-atmosphere was simulated by a Neumann boundary condition with a flux equal to $0.5 \mathrm{~mm} /$ day. The heating was modelled by imposing a heat flux equal to $55 \mathrm{~W} / \mathrm{m}^{2}$. This flux was imposed in the interface between the cables and the concrete.

\section{Sensitivity study for $\eta$ values of the surrounding soil}

The thermal vapour diffusion enhancement factor $(\eta)$ varies with the variation of the water content. To quantify the effect of the variation of this parameter on the moisture and heat transfers, two models were compared. The initial conditions, boundary conditions and material characteristics were identical for the two models and the only difference was the $\eta$ value of the surrounding soil. For one model $\eta$ was equal to 1 (dry soil), and for the other, it was equal to 5 (moderately moist soil). The heating period was fixed to 180 days.

The degree of saturation $(S)$ after 180 days of heating for the two values of $\eta$ is presented in Figure 3. In order to focus on the soil behaviour in the vicinity of the trench, the profiles are shown for $5 \mathrm{~m}$ in each direction from the centre of the trench. We can see in this figure the variation of the profiles of the degree of saturation as a result of heating. The maximal drying was occurred at the interface soil-concrete and the final degree of saturation was $48.3 \%$ and $38.9 \%$ for $\eta$ equal to 1 and 5 respectively. The global drying compared to the soil-atmosphere interface is related to the evaporation imposed at this boundary, letting moisture transfer to the outside of the system $(0.5 \mathrm{~mm} /$ day). Approaching the water table $(4 \mathrm{~m}$ of depth) the heating effect on the degree of saturation decreased.

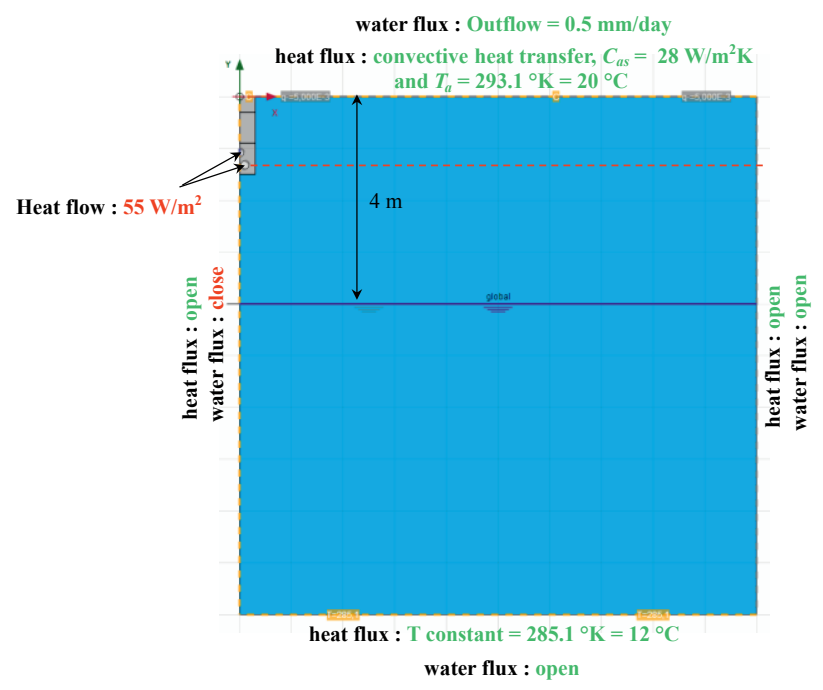

Figure 2. Model boundary conditions.

To study the radius of influence of the power cable, the degree of saturation $(S)$ after 180 days of heating is drawn throughout the dotted red line in Figure 4 . The initial degree of saturation was $80 \%$. At the contact with the trench, $S$ decreased to $48.9 \%$ for $\eta=1$ while it decreased to $39.5 \%$ for $\eta=5$. The larger decrease with $\eta=5$ is related to the role of this parameter in vapour transfer: Increasing $\eta$ accelerates the transfer of vapour resulting in a greater drying of the soil on one hand and a larger radius of influence on the other hand. The radius of influence is about $1.60 \mathrm{~m}$ for $\eta=1$ but it is about $3 \mathrm{~m}$ for $\eta=5$ (in addition to general drying caused by evaporation at the soil-atmosphere interface). 

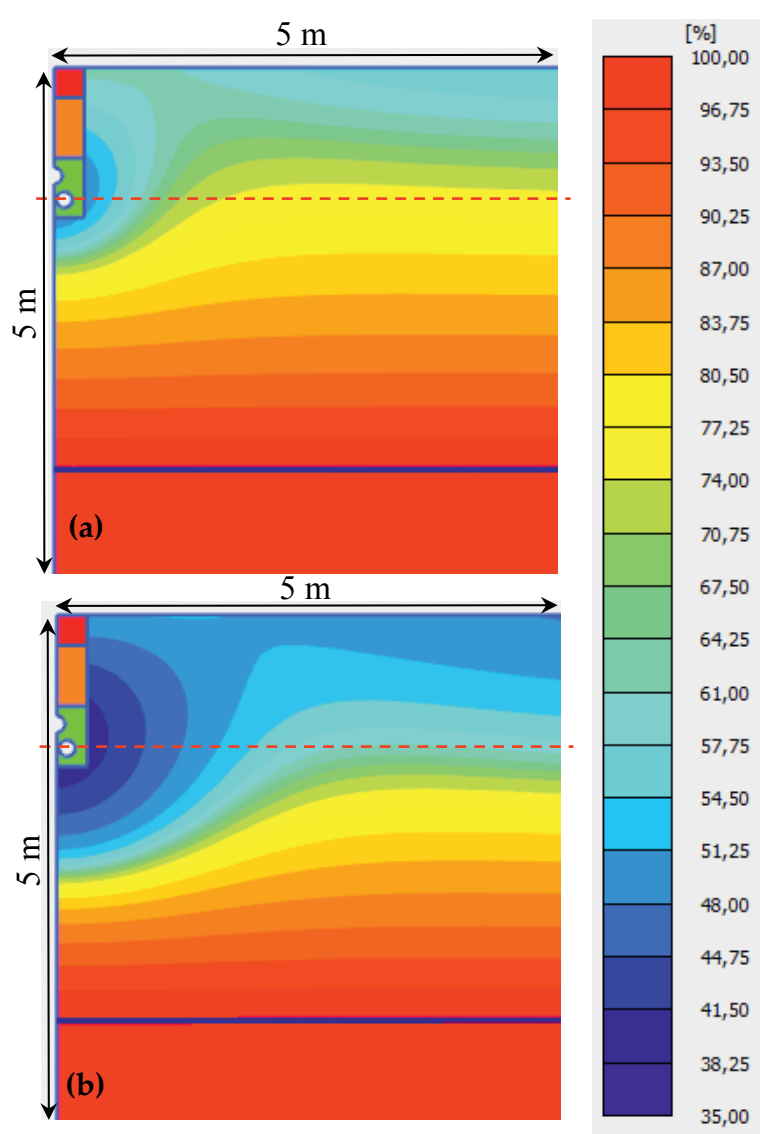

Figure 3. Degree of saturation after 180 days of heating: (a) $\eta=1$; (b) $\eta=5$.

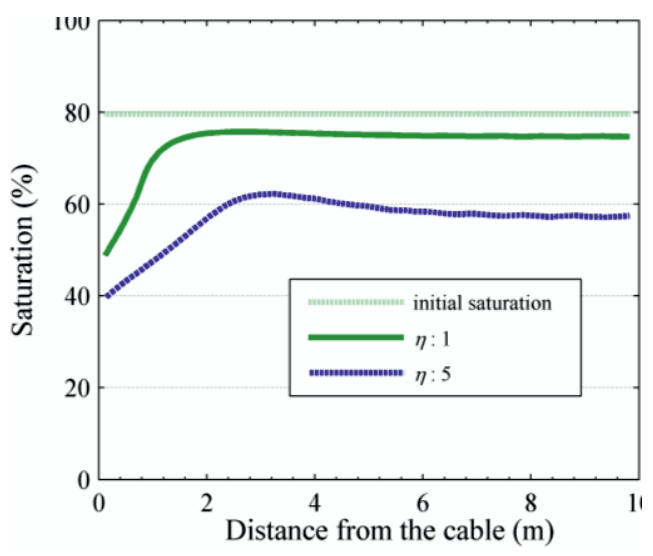

Figure 4. Degree of saturation after 180 days of heating throughout the dotted red line.

Figure 6 shows the temperature as a function of distance from the cable after 180 days. The heat propagation is almost the same. The maximal temperature was $49.5^{\circ} \mathrm{C}$ and $48.3^{\circ} \mathrm{C}$ for $\eta=1$ and $\eta=5$ respectively.

In order to study the kinetic of the temperature and degree of saturation variations, these parameters are drawn as a function of time for the point located at the right bottom of the trench $(0.30,-1.50)$ (Figure 6). For temperature, the variation is identical in the first 25 days and then there is a small difference between two curves, reaching $1.3{ }^{\circ} \mathrm{C}$ of difference after 180 days with $31{ }^{\circ} \mathrm{C}$ for $\eta=1$ and 29.7 for $\eta=5$. For degree of saturation, when $\eta=5$ there was an important decrease for the first
10 days and it was stabilized after 120 days. When $\eta=1$, the decrease of $S$ in the first days was less important than that of $\eta=5$ but it continued to decrease with almost the same rate until 50 days. After 180 days it was not completely stabilized. The dependence of $S$ variation to $\eta$ is related to the diffusion of water vapour through the soil under thermal gradient (equation (3)). The thermal vapour diffusion coefficient, $D_{T V}$, depends also on $\eta$ and the increasing of $\eta$ increases this parameters. The higher the value of $D_{T v}$, the higher the vapour transfer induced by temperature gradient. Consequently the decrease of the degree of saturation is more important, and the soil will be drier in that case.

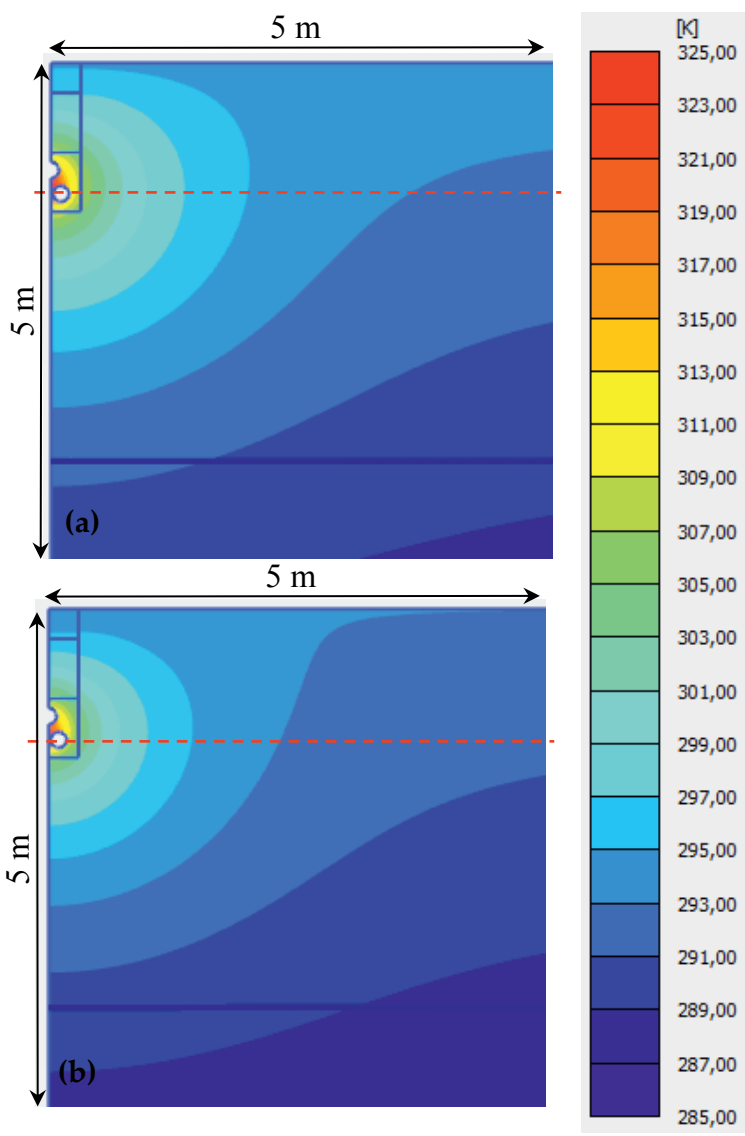

Figure 5. Temperature after 180 days of heating: (a) $\eta=1$; (b) $\eta=5$.

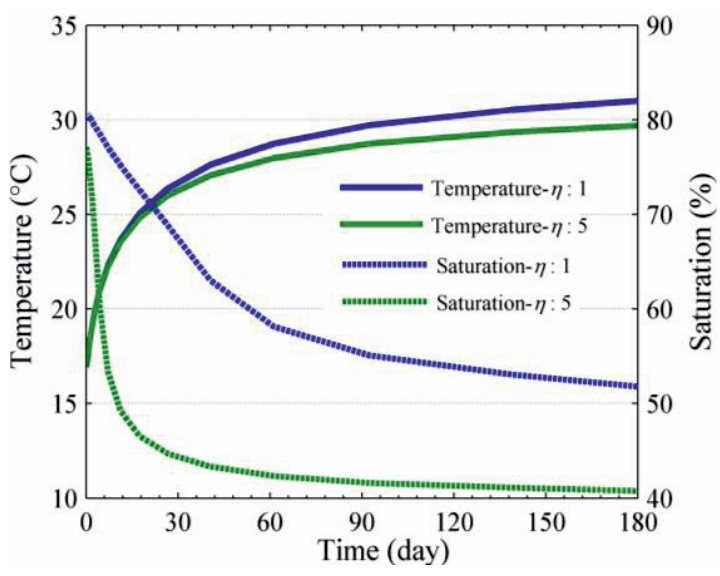

Figure 6. Temperature and saturation as a function of time for the point located at the right bottom of the trench. 


\section{Conclusion}

The main objective of this work was to highlight the coupled heat and moisture flows around a buried electrical cable and quantify the effect of thermal vapour diffusion enhancement factor on this behaviour. The key parameters of this modelling were on one hand, the conventional parameters (the thermal conductivity, thermal capacity, retention curve and the permeability coefficient) and the diffusivity of water vapour $\left(D_{v}\right)$ and on the other hand the thermal vapour diffusion enhancement factor $(\eta)$. The increase of $\eta$ resulted in a more significant drying and in a less important and slower temperature increase. This shows the high effect of transfer in vapour phase in the soil surrounding buried electrical cables. We observed also the dependence of the influence radius to the values of $\eta$. In this study, the energy used to evaporate water and the heat transported by vapour are not considered. Taking into account this part of energy balance by using the evaporation heat latent of water in the equations will increase the heat.

\section{Acknowledgment}

This study was part of the VALO CQFD research project funded by ADEME.

\section{References}

1. De Leon F, Anders GJ (2008) Effects of Backfilling on Cable Ampacity Analyzed With the Finite Element Method. IEEE Trans Power Deliv 23:537-543.

2. de Lieto Vollaro R, Fontana L, Vallati A (2011) Thermal analysis of underground electrical power cables buried in non-homogeneous soils. Appl Therm Eng 31:772-778.

3. Hanna MA, Chikhani AY, Salama MMA (1998) Thermal analysis of power cable systems in a trench in multi-layered soil. IEEE Trans Power Deliv 13:304-309.

4. Adams JI, Baljet AF (1968) The thermal behavior of cable backfill materials. IEEE Trans Power Appar Syst PAS-87:1149-1161.

5. El-Kady MA (1982) Optimization of Power Cable and Thermal Backfill Configurations. IEEE Trans Power Appar Syst PAS-101:46814688.

6. Mitchell JK, Abdel-hadi ON (1979) Temperature Distributions around Buried Cables. IEEE Trans Power App Syst 98:1158-1166.

7. Gela G, Dai JJ (1988) Calculation of thermal fields of underground cables using the boundary element method. IEEE Trans Power Deliv Vol 3, No 4, Oct 3:1341-1347.

8. Bernath A, Olfe DB, Ferguson BW (1984) Heat transfer measurements on uniqually loaded underground power cables with constant and cyclic currents. IEEE Trans Power Appar Syst PAS-103:2799-2806.

9. Al-Saud M s., El-Kady M a., Findlay RD (2006) Accurate Assessment of Thermal Field and Ampacity of Underground Power Cables. In:
Can. Conf. Electr. Comput. Eng. pp 651-654.

10. Kellow MA (1981) A numerical procedure for the calculation of the temperature rise and ampacity of underground cables. IEEE Trans Power Appar Syst PAS-100:3322-3330.

11. Philip JR, De Vries D a. (1957) Moisture movements in porous materials under temperature gradients. Trans Am Geophys Union 38:222-232.

12. Gurr CG, Marshall TJ, Hutton JT (1952) Movement of water in soil due to a temperature gradient. Soil Sci 74:335-346.

13. Taylor SA, Cavazza L (1954) The movement of soil moisture in response to temperature gradients. Soil Sci Soc Am J 18:351-358.

14. Cass A, Campbell GS, Jones TL (1984) Enhancement of thermal water vapor diffusion in soil. Soil Sci Soc Am J 48:25-32.

15. Bonsu M (1997) Soil water management implications during the constant rate and the falling rate stages of soil evaporation. Agric Water Manag 33:87-97.

16. Hiraiwa Y, Kasubuchi T (2000) Temperature dependence of thermal conductivity of soil over a wide range of temperature $(5-75 \mathrm{C})$. Eur J Soil Sci 211-218.

17. Wildenschild D, Roberts JJ (2001) Experimental tests of enhancement of vapor diffusion in Topopah Spring Tuff. J Porous Media 4:1-13.

18. Cary JW (1979) Soil Heat Transducers and Water Vapor Flow1. Soil Sci Soc Am J 43:835.

19. Jury WA, Letey J (1979) Water vapor movement in soil: reconciliation of theory and experiment. Soil Sci Soc Am J 43:823-827.

20. Haxaire A, Galavi V, Brinkgreve RBJ (2013) Definition and implementation of a fully coupled THM model for unsaturated soils. In: Kwasniewski M, Lydzba D (eds) Rock Mech. Resurces, Energy Environ. Taylor \& Francis Group, Wroclaw, Poland, pp 507-512

21. Milly PCD (1984) A Simulation Analysis of Thermal Effects on Evaporation From Soil. Water Resour Res 20:1087.

22. Kimball B a., Jackson RD, Reginato RJ, et al. (1976) Comparison of Field-measured and Calculated Soil-heat Fluxes1. Soil Sci Soc Am J 40:18.

23. Lu S, Ren T, Yu Z, Horton R (2011) A method to estimate the water vapour enhancement factor in soil. Eur J Soil Sci 62:498-504.

24. Sakai M, Toride N, Šimůnek J (2009) Water and Vapor Movement with Condensation and Evaporation in a Sandy Column. Soil Sci Soc Am J 73:707.

25. Heitman JL, Horton R, Ren T, et al. (2008) A Test of Coupled Soil Heat and Water Transfer Prediction under Transient Boundary Temperatures. Soil Sci Soc Am J 72:1197. doi: 10.2136/sssaj2007.0234

26. Garzoli KV, Blackwell J (1987) An analysis of the nocturnal heat loss from a double skin plastic greenhouse. J Agric Eng Res 36:75-86. 